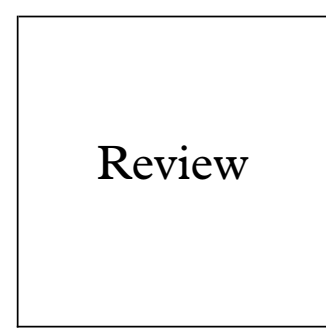

\title{
Sexually transmitted diseases in sexually abused children: medical and legal implications
}

\author{
Margaret R Hammerschlag
}

Sexually transmitted diseases (STDs) may be transmitted during sexual assault. In children, the isolation of a sexually transmitted organism may be the first indication that abuse has occurred. Although the presence of a sexually transmissible agent from a child beyond the neonatal period is suggestive of sexual abuse, exceptions do exist. In this review I discuss the issues of the transmissibility and diagnosis of STDs in the context of child sexual abuse. Rectal or genital infection with Chlamydia trachomatis among young children may be the result of perinatally acquired infection and may persist for as long as 3 years. A major problem with chlamydia testing in the context of suspected sexual abuse in children has been the inappropriate use of non-culture tests. Although the new generation of nucleic acid amplification tests have shown high sensitivity and specificity with genital specimens from adults, data on use of these tests on any site in children are practically non-existent. Bacterial vaginosis (BV) has been identified among children who have been abused and among those who have not been abused. However, many of the methods used to diagnose BV in adults have not been evaluated in children. Recent studies of perinatal infection with human papillomavirus (HPV) have been inconclusive. HPV DNA has been detected at various sites in children who have not been abused. The relation to the development of clinically apparent genital warts is unclear. Although HIV can be acquired through sexual abuse in children, the exact risk to the child and which children should be screened is still controversial. (Sex Transm Inf 1998;74:167-174)

Keywords: STDs; child sexual abuse; Chlamydia trachomatis; gonorrhoea; human papillomavirus

Sexual assault is a violent crime that affects men, women, and children of all ages. Sexually transmitted diseases (STDs) may be transmitted during sexual assault. In children, the isolation of a sexually transmitted organism may be the first indication that abuse has occurred. However, most sexually abused children do not present with genital complaints. Although the presence of a sexually transmissible agent from a child beyond the neonatal period is suggestive of sexual abuse, exceptions do exist.

The incidence and prevalence of sexual abuse of children are difficult to estimate, mainly because much of the sexual abuse in childhood escapes detection. There have been several relatively extensive studies of sexual abuse of children in the United States that have examined sex, race, and age dependent variables. ${ }^{1-4}$ Patterns of childhood sexual abuse appear to depend on the sex and age of the victim. About $80 \%$ to $90 \%$ of abused children are female, with mean ages of 7-8 years. Most, $75 \%$ to $85 \%$, were abused by a male assailant, adult or minor known to the child. This individual is most likely to be a family member, especially the father or father substitute (stepfather, mother's boyfriend), uncles, and other male relatives. Victims of stranger or unknown assailants tend to be older than the children abused by a known person and usually involve only a single episode of abuse. In contrast, abuse of family members or acquaintances usually involves multiple episodes over periods of time ranging from a week to years.

Most victims describe a single type of sexual activity, but over $20 \%$ have experienced multi- ple types of forced sexual acts. Vaginal penetration has been reported to occur in approximately half and anal penetration in one third of female victims. Over half of male victims have experienced anal penetration. Other types of sexual activity include orogenital contact in $20 \%$ to $50 \%$ of victims and fondling. Children who are abused by a known assailant usually experience less trauma than victims of assault by a stranger.

\section{Risk of infection}

An accurate determination of the risk of sexually transmitted conditions in victims of sexual abuse has been hindered by a variety of factors: (i) The prevalence of sexually transmitted infections may vary regionally and among different populations within the same region. (ii) Few studies have attempted to differentiate between infections existing before the abuse. This may be impossible in settings where the abuse has been chronic (of long duration). The presence of pre-existing infection in adults is usually related to earlier sexual activity. In children pre-existing infection may be related to prolonged colonisation after perinatal acquisition, inadvertent non-sexual spread, prior peer sexual activity, or prior sexual abuse. (iii) The incubation periods for STDs range from a few days for Neisseria gonorrhoeae to several months for human papillomavirus. The incubation periods and timing of an examination after an episode of abuse are critically important in detecting infections. Multiple episodes of abuse have been found to increase the risk of infection probably by increasing the number of 
contacts with an infected individual. In most cases, the site of infection is consistent with the child's history of assault. Rates of infection also will vary with respect to the type of assault initially described. Vaginal or rectal penetration is more likely to lead to detectable infection, whereas fondling is unlikely to lead to infection. However, the majority of children who are abused will have no physical complaints related to either trauma or infection. I have not covered the issue of fomites. Although this has been proposed there are no data to support this in adults or children.

\section{Gonorrhoea}

Gonorrhoea is the most frequent STD found in abused children, reported to occur in $1-30 \%$ of abused children. ${ }^{5}$ Infection is frequently asymptomatic, especially in the rectum and pharynx. The isolation of $N$ gonorrhoeae from a prepubertal child is probably the most useful indicator of sexual abuse or activity. It has generally been recommended that all children suspected of being sexually abused have cultures obtained from genitals, rectum, and pharynx. ${ }^{6}$ This came in part because of the high predictive value of the presence of gonococcal infection and the occurrence of sexual abuse and several studies which suggested that many of these infections were asymptomatic, even vaginal infections. ${ }^{7}$ As recent studies have found the rates of gonococcal infection in abused children to be $<3 \%,{ }^{8-10}$ it has been suggested that selective criteria could be used to determine which children suspected of being sexually abused should be screened rather than screening every child to avoid the trauma and expense of obtaining unnecessary cultures. In 1991 the American Academy of Pediatrics (AAP) Committee on Child Abuse and Neglect suggested that routine cultures and screening of all children for gonorrhoea was not necessary or recommended because the yield of positive cultures was very low in asymptomatic prepubertal children, especially those with histories of fondling only. ${ }^{11}$ The AAP suggested that testing be done when "epidemiologically indicated or when the history and/or physical findings suggest the possibility of oral, genital or rectal contact". ${ }^{11}$

In 1995, Seigel et al applied a modification of these criteria to children being evaluated for suspected sexual abuse at the Children's Hospital Medical Center in Cincinnati. ${ }^{9}$ They were as follows: the victim has a history of genital discharge or contact with the perpetrator's genitalia, the victim has a genital discharge or trauma on examination, the victim has a history of consensual sexual activity, the victim was post pubertal (Tanner III), the victim had sexual contact with a perpetrator known to have an STD, or there was a family member with an STD. ${ }^{9}$ They evaluated 855 children over a 1 year period; there were 704 girls and 151 boys. Of 379 girls tested for gonorrhoea 12 (3.2\%) were positive; all had either vaginal discharge or a history of vaginal penetration. All the prepubertal girls had symptomatic vaginal discharges. Subsequently, Ingram et al ${ }^{10}$ evaluated these criteria to determine if they would have accurately detected all cases of vaginal gonococcal infection in their population. Of 2898 girls seen over a 10 year period, $2731(94 \%)$ had vaginal cultures obtained for $N$ gonorrhoeae; $84(3.1 \%)$ had positive cultures, 80 of whom had a vaginal discharge. The four remaining girls who were asymptomatic included two with a history of vaginal intercourse with an alleged perpetrator with gonorrhoea, one who had $N$ gonorrhoeae isolated from her urine culture, and one whose preteenage sister had gonorrhoea. Thus all of the 84 girls with vaginal gonorrhoea would have been identified using the selective screening criteria recommended by the AAP.

Although it appears that one can use these selective criteria for vaginal specimens in girls, there are insufficient data to support their use for rectal and pharyngeal infection or genital infections in boys. Rectal and pharyngeal infections with $N$ gonorrhoeae are more likely to be asymptomatic in children. ${ }^{72}$

\section{Chlamydia trachomatis}

Infections caused by $C$ trachomatis are the most prevalent STDs in adults in the United States today, over three million cases a year being reported. ${ }^{13}$ Most infections in adults are asymptomatic. Sexually active adolescents have the highest rates of infection, often $>20 \%$, and are at highest risk for serious sequelae - that is, pelvic inflammatory disease. ${ }^{14} C$ trachomatis can cause prolonged asymptomatic infection in adults and children.

Infection in children can be perinatally acquired. The risk of transmission from an infected mother to her infant at delivery is approximately $50 \% .{ }^{15}$ The infant may become infected at one or more anatomical sites including the conjunctiva, nasopharynx, rectum, and vagina. The most frequent clinical disease is neonatal conjunctivitis (inclusion conjunctivitis), which occurs in $20 \%$ to $50 \%$ infants born to infected mothers. Over half of these babies will also be infected in the nasopharynx; $10 \%$ to $20 \%$ of infants born to infected mothers will also be infected in the rectum, vagina, or both. ${ }^{15}$ Perinatally acquired rectal or vaginal infection is asymptomatic and may persist for at least 3 years. ${ }^{16}$

$C$ trachomatis rectal or vaginal infection in sexually abused children is relatively infrequent with prevalences of less than $5 \% .{ }^{5}$ These infections are usually asymptomatic. An important development in the management of $C$ trachomatis infections has been the advent of non-culture tests, which has greatly facilitated the screening and treatment of these infections in adults. However, in children suspected of being sexually abused, culture is still the preferred method of diagnosis. Non-culture antigen detection tests such as enzyme immunoassays (EIA) and direct fluorescent antibody (DFA) tests and DNA probes are not specific at rectogenital sites in children and are not approved for use at these sites. Use of these assays for the diagnosis of $C$ trachomatis infection in children suspected of being sexually abused has been associated with a significant number of false positive results. ${ }^{17-20}$ 
A new generation of nucleic acid amplification tests including polymerase chain reaction (PCR), ligase chain reaction (LCR), and transcription mediated amplification (TMA) are now available. These assays often have sensitivities that exceed that of culture while maintaining specificity. ${ }^{21} 22$ These assays are also sufficiently sensitive for detection of $C$ trachomatis in urine from men and women. ${ }^{23}$ Nucleic acid amplification methods are not approved for rectal specimens in adults nor any site in children including the rectum and vagina. Currently PCR (Amplicor, Roche Diagnostic Systems) is being evaluated for this indication in Canada. Preliminary data comparing culture and EIA with PCR in vaginal wash specimens from 35 children found a sensitivity of $100 \%$ and a specificity of $89.7 \%$ for PCR compared with culture. ${ }^{25}$ PCR was performed retrospectively on the culture specimens. Another study examined 258 specimens from males and females, but the anatomical sites were not specified ( $\mathrm{R}$ Peeling, personal communication). The sensitivity and specificities of PCR compared with culture were $66.7 \%$ and $99.6 \%$, respectively. All the positive PCR results were confirmed with another PCR reaction targeting the major outer membrane gene using the original culture specimen. The culture positive, PCR negative specimen in the second study was a rectal specimen. The investigators reported that many rectal specimens contained inhibitors that could not be removed by dilution or phenol chloroform extraction. PCR is not approved for rectal specimens from adults; the major reason is the frequent occurrence of inhibitors of DNA polymerase in faecal material. This would adversely affect the sensitivity of the test.

False positive results can also occur with PCR and LCR as a result of amplicon carryover. This is minimised with the current commercially available assays but can still occur. As described above, there are practically no data on the performance of PCR or LCR at these sites in children. Recent studies on use of PCR in cervical specimens in adult women have reported poor reproducibility with some specimens. ${ }^{26}$

The sensitivity of the nucleic amplification tests for detection of $C$ trachomatis in urine may be an attractive application for children as a non-invasive specimen is used. Obviously, before these assays could be used for this indication, studies comparing these tests with vaginal and urethral cultures from children for $C$ trachomatis would need to be undertaken.

Chlamydial culture should be isolation in tissue culture and confirmation by microscopic identification of the characteristic intracytoplasmic inclusions after staining with a species specific fluorescein conjugated monoclonal antibody. Use of genus specific antibodies can lead to confusion with $C$ pneumoniae in respiratory specimens. ${ }^{27}$ EIAs should not be used for culture confirmation as they can cause false positives. ${ }^{20}$ There is no standardisation or regulation of chlamydial culture methods at this time in the United States and most other countries. ${ }^{28}$ In Canada, approved reference laboratories are designated for performance of cultures for forensic purposes. With the expanding role of large commercial laboratories in the United States a problem now encountered by many clinicians is lack of control over what type of chlamydia test is actually done. Although the paediatrician may request a culture, the laboratory may unilaterally decide to use a non-culture test, often as a cost saving measure and because they do not fully understand the legal implications.

\section{Syphilis}

Children can be infected with Treponema pallidum either in utero (congenital syphilis) or in a manner similar to adults (acquired syphilis). Although congenital syphilis is far more common than acquired syphilis the emphasis is on acquired syphilis. Congenital syphilis is transmitted transplacentally from an infected mother to her fetus; it is not spread by sexual transmission. In contrast, acquired syphilis in children is almost always sexually transmitted from abuse by an infected adult. ${ }^{29-31}$ Almost all preadolescent children with syphilis have acquired syphilis from adults; thus, the epidemiology of adult syphilis is important in understanding syphilis in children. The epidemiology of both congenital and acquired syphilis in children is really the epidemiology of syphilis in adults. Syphilis in adults is usually associated with lower socioeconomic status, race (black > hispanic $>$ white), and living in an urban area.

Syphilitic chancres in extragenital sites may possibly be from non-sexual contact, ${ }^{32}$ but it is unwise to dismiss sexual abuse as a cause of transmission of syphilis, even when there is no specific history of abuse given by the child. ${ }^{31}$ The prevalence of syphilis among children suspected of having been sexually abused is lower than some other STDs such as gonorrhoea. The prevalence of positive serology for syphilis in six surveys of abused children published from 1988 to the end of 1992 ranged from 0 to $1.8 \% .^{5833}$ These numbers indicate that syphilis is not very common among children who have been abused, and is seen less often than it was in the preantibiotic era. ${ }^{29}$ However, syphilis continues to be a prominent STD among abused children in developing countries. Pandhi $e t a l^{34}$ found evidence of syphilis in $27.6 \%$ of symptomatic abused children referred to a dermatology and STD department in Delhi, India.

Although routine serological testing for syphilis has been recommended in the evaluation of children who are sexually abused, this may in fact, not be done by many practitioners. Bays and Chadwik ${ }^{35}$ distributed a questionnaire to medical personnel attending a special session on child abuse at the annual meeting of the AAP in 1986 and a 3 day seminar on child sexual abuse in 1987 in San Diego. They received replies from 82 respondents from 24 states plus the District of Columbia and Canada. Only one third of the responders routinely tested all sexually abused children for syphilis. The major reasons cited for not routinely testing were trauma and low yield. The remaining respondents used a variety of 
selective criteria to determine which children to test. However, the rate of syphilis among the children who were screened in this study was 49 cases/100 000, which was comparable with that of adults living in a high prevalence area. Most of these children were asymptomatic. The consequences of undetected, untreated primary syphilis in children are not known; the risk may be low, but the consequences are potentially devastating. Almost all will remain fluorescent treponemal antibody positive and at least one third will eventually develop symptomatic secondary syphilis. Some children with undetected syphilis might be treated later if they develop signs of secondary syphilis or if they have positive serology after routine premarital or prenatal testing. Some may be treated with antibiotics incidentally for other indications. Although the $\mathrm{CDC}^{6}$ recommends an 8 week follow up visit for sexually abused children with a repeat of serological testing, it is not known how frequently this is actually done. The risk of a sexually abused child acquiring syphilis and ultimately escaping detection and treatment is not known.

Bays and Chadwick ${ }^{35}$ suggested the following minimum criteria for selective testing of sexually abused children for syphilis: (1) children with evidence of STDs; (2) adolescents; (3) foreign born children; (4) children with a parent, family member, or perpetrator with syphilis; (5) children living in areas with a high incidence of syphilis.

\section{Human papillomavirus, genital warts, condylomata acuminata}

Human papillomavirus (HPV) is probably the most common viral sexually transmitted disease in the United States today. There is a large asymptomatic reservoir. Reliance on the presence of visible condylomata greatly underestimates the prevalence of this infection. Newer methods for detection of HPV DNA include dot blot hybridisation, Southern blotting, and modifications of PCR. HPV DNA has been found in cervices of up to $35 \%$ of adolescent women, a minority of these women have clinically apparent warts. ${ }^{36} 37$ Condylomata have been reported in 1-2\% of abused children and $50 \%$ to $75 \%$ of cases of genital warts in children reported in the literature appear to be the result of abuse. ${ }^{5}$ Like $C$ trachomatis, HPV can be acquired perinatally, leading to juvenile laryngeal papillomatosis and genital warts; however, data on the risk of transmission are limited.

The data on the prevalence of genital HPV among pregnant women are limited. Fife and colleagues $^{38}$ detected HPV DNA in cervical scrape specimens from $11 \%$ of unselected pregnant women presenting to an inner city obstetric service in the first trimester. Only two of the 26 women with positive specimens had genital warts. Rando and colleagues ${ }^{39}$ found HPV DNA in $20.9 \%$ of exfoliated cervical cells from pregnant women during the first trimester. The prevalence dramatically increased to $46 \%$ during the third trimester, but decreased to $17.5 \%$ of women post partum. A recent study by Watts et $a l^{40}$ from Seattle found that
$74 \%$ of pregnant women had historical, clinical, or DNA evidence of genital HPV infection during pregnancy.

Studies of transmission and infection with genital HPV types during the neonatal period have given highly variable results. In 1989, Sedlacek and colleagues ${ }^{41}$ detected HPV DNA by Southern blot in neonatal nasopharyngeal aspirates from 11 of $23(47.8 \%)$ infants born by spontaneous vaginal delivery to women who had HPV DNA present in their cervical scrapings. HPV DNA was also detected in the nasopharyngeal washes of four of $18(22 \%)$ infants born to HPV negative women. HPV was not detected in two infants of positive women who were delivered by caesarean section. Using similar methods, Fife et $a l^{42}$ demonstrated HPV DNA in oral cavity scrapings and foreskins from five of $11(45.5 \%)$ newborn infants whose mothers were known to have HPV DNA present in the cervix during pregnancy. A subsequent study by Pakarian et $a l^{43}$ found HPV DNA in $10(50 \%)$ of 20 oral specimens from infants born to HPV positive mothers and one $(19 \%)$ of 11 infants born to negative mothers evaluated at 1 day of age. In contrast, HPV DNA was detected genital specimens obtained at 6 weeks of age from six $(30 \%)$ of 20 infants born to HPV positive mothers compared with two $(18 \%)$ of 11 infants born to negative mothers. The frequent detection of HPV DNA from infants within a few days of birth may represent transient mucosal colonisation. This is suggested by the findings of Pakaraian et $a l^{43}$ where the prevalence of HPV DNA among infants born to positive mothers decreased at 6 weeks of age. Conversely, the high presence of HPV DNA in infants born to negative women suggests contamination.

Data on the presence of HPV DNA in children beyond the neonatal period are also inconsistent. The results of three such studies found the presence of HPV DNA in children born to infected mothers to range from $1.2 \%$ to $77 \%{ }^{404445}$ The rate of HPV infection in children born to uninfected women ranged from $1 \%$ to $50 \%$. The anatomical sites tested varied from study to study and included oral, genital, and rectal specimens. Similarly, the duration of follow up also differed from study to study. Puranen et $a l^{44}$ examined children, 4 months to 11.3 years of age, but each child was sampled only once. Cason et $a l^{45}$ evaluated infants born to positive and negative women at 1 day, 6 weeks, and 6 months of age. Watts et $a l^{40}$ evaluated the children in their study at birth, 6 weeks, and $6,12,18,24$, and 36 months of age. The high rate of HPV DNA, $50 \%$, detected in infants born to negative mothers in the study of Cason et al ${ }^{45}$ suggests the possibility of horizontal transmission between infants and care givers.

However, the relation between the presence of HPV DNA and the ultimate development of disease in these children is not clear. None of the infants followed by Watts et $a l^{40}$ developed any clinical manifestations of HPV infection, specifically genital warts or laryngeal papillomatosis. Puranen et $a l^{44}$ found minor hyperplastic 
growths in the oral mucosa of $21(21 \%)$ of 98 children, but HPV DNA was detected in only one papilloma (site not specified) in one child. It is possible that the duration of follow up in the study of Watts et $a l^{40}$ was not long enough. However, in juvenile onset laryngeal papillomatosis (clinical onset before puberty), $75 \%$ of cases are diagnosed before 5 years of age. The types of HPV involved are the same types responsible for the majority of genital warts, HPV-6 and HPV-11. Juvenile onset laryngeal papillomatosis is very rare, Shah and colleagues ${ }^{46}$ calculated on the basis of crude estimates of the number of children born to infected mothers and of new cases of juvenile onset disease, the risk of developing disease for a child born to an infected mother was one in several hundred exposures. It is felt that most genital warts in children less than 3 years of age are most probably due to perinatal acquisition. The results of these studies of perinatal transmission casts some doubt on this. DNA typing in three studies of genital condylomata in children reported $10-20 \%$ to have skin HPV types (HPV-2 and 3). ${ }^{47-49}$

Gutman et $a l^{50}$ examined vaginal washes from sexually abused girls and found HPV DNA in five of 15 (33.3\%) (HPV-6, 11, 15) compared with none of 17 control children. Results of the wash did not correlate with presence or absence of external anogenital warts in these children. This was a very small sample and the cases were older than the control children, with a median age of 59 months (range 23-131 months) for the cases versus a median age of 34 months (range 3-68 months) for the controls.

In a subsequent study the same investigators examined cervical vaginal wash specimens and biopsies from 18 girls with external genital warts. ${ }^{51}$ They found HPV-6, HPV-11, and HPV-16 DNA in biopsies of 17 of the external lesions and HPV-6 and HPV-16 in cervical vaginal wash specimens from seven of these children, confirming that as has been found with adults, young girls with external anal genital warts are also frequently infected with HPV at internal mucosal sites. The children ranged from 1 to 10 years of age with a median of 4.8 years. A confirmed history of sexual abuse was obtained from seven of nine children over 3 years of age but could not be confirmed for any of the nine children 3 years of age or younger; eight were considered "unknown" and one probable. The authors assumed that their abuse status was unknown because they were preverbal. However, the possibility of perinatal acquisition was not discussed. Considering that many cases of juvenile onset laryngeal papillomatosis do not clinically manifest before several years of age, the same should apply to genital warts in these children. To confuse the issue further, Jenison et $a l^{52}$ found HPV-6 and HPV-16 DNA in $24 \%$ and $19 \%$, respectively, of oral mucosal scrapings from 21 healthy prepubertal children recruited from an urban preschool in Seattle. The prevalence was similar to that found in 35 healthy adult men: $17 \%$ had HPV-6 and $23 \%$ had HPV-16 present in their mucosal scrapings. There was also no difference between the prevalence of serum antibody to HPV-6 and HPV-16 in adults and children. The authors concluded that asymptomatic infection with these HPV types are relatively common and that acquisition of these HPV types may occur by modes other than sexual transmission. Whether childhood infection was a consequence of perinatal transmission or by family or environmental contact would need to be determined. At this time, the data on the epidemiology of the acquisition of clinically apparent HPV disease in children are inconclusive.

\section{Bacterial vaginosis}

Bacterial vaginosis (BV) is the most frequent cause of vaginal discharge in adults and probably also in children. ${ }^{12}{ }^{53} \mathrm{BV}$ is a disorder of the vaginal flora characterised by a decrease in hydrogen peroxide producing lactobacilli, an increase in anaerobes (Mobiluncus spp, Bacteroides spp), Gardnerella vaginalis, and $\mathrm{Myco-}$ plasma hominis..$^{53} G$ vaginalis is not the sole cause of BV and is not a suitable marker of sexual activity. The organism can be found in asymptomatic and virginal girls. The diagnosis of $\mathrm{BV}$ is not made by culturing for $G$ vaginalis, but by examining a vaginal wet mount specimen for the presence of clue cells (vaginal epithelial cells studded with small Gram negative rods), a positive amine or "whiff" test (the development of a fishy odour after addition of potassium hydroxide to the vaginal fluid) and, in adults, a vaginal $\mathrm{pH}>4.5$. Recent studies have suggested that a reliable, reproducible diagnosis of $\mathrm{BV}$ can be made by examining Gram stained vaginal smears. ${ }^{54}{ }^{55}$ The smear is examined for the presence of epithelial cells and the following morphotypes: lactobacilli (Gram positive rods), $G$ vaginalis or bacteroides (small Gram variable rods or Gram negative rods), and curved Gram positive rods. A smear containing no lactobacilli and heavy Gram negative and/or Gram variable rods and curved Gram positive rods would be diagnostic for BV. This method appears to be more sensitive and specific for the diagnosis of BV than the combination of clue cells, whiff test, and $\mathrm{pH}$ in adults; however, it has not been evaluated in prepubertal children. ${ }^{54} 55$

Studies in adults and adolescents have found $\mathrm{BV}$ and trichomoniasis to be the most transmissible infections after sexual assault, approximately $15 \%$ of victims acquire these infections. ${ }^{56}{ }^{57}$ Data on the prevalence of $\mathrm{BV}$ in children with vaginal discharge and suspected sexual abuse are limited. The majority of case reports and studies in children have been based only on identification of $G$ vaginalis, not on examination of a wet mount for clue cells, or whiff test. ${ }^{58} G$ vaginalis has been isolated from vaginal cultures of $1 \%-32 \%$ normal or control children, compared with $7 \%-34 \%$ in sexually abused or sexually active girls. ${ }^{59-61}$ Presence of $G$ vaginalis was generally not associated with vaginal discharge in these children. Using an examination of vaginal wash for clue cells and whiff test, Hammerschlag and colleagues ${ }^{62}$ demonstrated the development of BV in $13 \%$ of sexually abused girls compared with none of 
the controls. Ingram et $a l^{8}$ examined vaginal discharges from 99 children suspected of being sexually abused, seven had clue cells and/or a positive whiff test; $28 \%$ of the positive girls had a history of sexual contact. It is still not clear how often $\mathrm{BV}$ is a cause of vaginal discharge in children using appropriate diagnostic methods-specifically, examination of a wet mount of vaginal secretions or vaginal wash for clue cells, whiff test, or Gram stain. We also do not know how often BV is acquired after sexual assault in children, also using the above methods.

\section{Trichomonas vaginalis}

$T$ vaginalis is a common cause of vaginitis in adults. Along with BV, it is one of the most frequent infections acquired after sexual assault in adult and adolescent women. ${ }^{56}$ Perinatal infection may occur with infection of the vagina, and presence of organisms in the urine, especially when "bagged" specimens are examined. Infection with $T$ vaginalis in these infants may persist for up to 1 year if not treated. Beyond infancy, presence of $T$ vaginalis in a vaginal specimen is strongly suggestive of sexual abuse. Very limited data are available about the risk of acquiring trichomoniasis after sexual abuse in children. In many studies only symptomatic children were tested, usually by examination of wet mounts of vaginal discharge. Diagnostic methods used is an issue, as microscopic examination of a wet mount of vaginal fluid is relatively insensitive and has a significant subjective component. We have all faced the telephone call from the laboratory reporting that one trichomonad was seen by one observer in a urine specimen collected for another purpose. There is a commensal species, Thominis, which is an inhabitant of the bowel and which can only be differentiated from $T$ vaginalis by the length of the undulating membrane. Culture, using several different media including Diamonds and modified thioglycolate, is more sensitive than examination of a wet mount, but has only been evaluated in adults. ${ }^{63}$

\section{Herpes simplex virus}

Genital herpes is increasing in prevalence among adults. Asymptomatic shedding in adults is now recognised as being frequent. Herpes simplex virus (HSV) can be acquired by children through sexual assault. ${ }^{5}$ Clinically the children usually present with ulcers and/or vesicles. Infection with HSV can also recur in these children. Children can also acquire genital infection by autoinoculation if they have gingivostomatitis. The type of virus (HSV-1 v HSV-2) does not rule out sexual abuse since $10 \%$ to $20 \%$ of genital herpes in adults can be due to type 1 . Commercially available serological assays cannot differentiate between antibody to HSV-1 or HSV-2, and thus are of little value forensically. However, the risk of acquisition of HSV after assault in children is unknown.

\section{Human immunodeficiency virus infection}

Human immunodeficiency virus (HIV) is now the major cause of mortality among young adults in the United States today. More of these infections are resulting from heterosexual transmission. As of 1997, over 5000 children with AIDS under 13 years of age have been reported to the CDC. HIV is predominantly transmitted to children from their mothers. ${ }^{64}$ The rates of perinatally acquired infection in the United States are decreasing, in part owing to use of prophylaxis with AZT in pregnant women and infants after delivery. Nearly all cases of HIV acquired by blood transfusions in the United States occurred before donor screening practices were implemented in March 1985. HIV infection can be acquired after sexual assault. Sexual abuse as a mode of HIV transmission is probably underestimated because its occurrence is difficult to ascertain. Gutman and colleagues ${ }^{65}$ reported that 14 $(14.6 \%)$ of 96 children with HIV infection being followed at her institution were confirmed to have been sexually abused. Sexual abuse was determined to be the only means of transmission in four of these children. Approaching the problem from another perspective, Gellert et al ${ }^{66}$ found that of approximately 5622 children who had HIV antibody tests obtained during assessment of suspected sexual abuse, $41(0.7 \%)$ were HIV seropositive. Of these 41, 28 were thought to have acquired HIV from the abuse, while 13 had other risk factors such as possible perinatal transmission. The most important confounding variable was maternal infection. The risk factors for HIV infection are similar to the risk factors for child sexual abuse-drug abuse and/or alcoholism in a parent, prostitution at home, poverty. Some cases of perinatally acquired HIV infection may not present with an AIDS defining illness until over 10 years of age. However, Seigel and colleagues ${ }^{67}$ described a case of a 12 year old girl who presented with Pneumocystis carinii pneumonia caused by unrecognised HIV infection resulting from sexual abuse. This child was well known to the child welfare system, having been removed from her home at 7 years of age. However, she continued to be abused by her natural father, presenting at 8 years of age with evidence if vaginal penetration and $C$ trachomatis infection. The sexual abuse by the father continued and the child was found to have trichomoniasis at 11 years of age after being raped by the mother's boyfriend. The child's mother was tested for HIV at that time and was negative. The authors of this report felt that this experience supports screening for HIV in sexually abused children whose risk factors include acquisition of another STD or multiple abusers.

There are no data as yet as to the risk of acquisition of HIV infection after sexual assault or abuse in children. Some investigators feel that we should be offering HIV testing to victims of sexual abuse, routinely or in selected situations. Unfortunately, this creates number of legal and ethical issues, especially in the United States, where parental informed consent 
is required for HIV testing. There are also legal issues in obtaining testing from the alleged perpetrator. Should we be offering prophylaxis in high risk situations (the perpetrator is HIV positive or at risk for HIV)? This may be relevant as we now have what appear to be very effective post-exposure prophylaxis regimens. However, prophylaxis would not be appropriate for most victims of child sexual abuse as the abuse is chronic, often over many months and years. A prospective study of the risk of HIV infection in children after sexual assault or abuse with an effort to define which children should be tested routinely is clearly indicated.

In conclusion, the presence of a sexually transmitted disease in a prepubertal child has both medical and legal implications. All sexually transmitted diseases do not have the same value forensically. It is important that the practitioner understand the epidemiology of these infections as well as which tests are appropriate for screening and diagnosis in children. Misdiagnosis of a sexually transmitted disease can be as devastating to the child and his or her family as failure to make the diagnosis.

1 Rimsza ME, Niggermann EH. Medical evaluation of sexually abused children: a review of 311 cases. Pediatrics 1982;69:8-15.

2 Jones JG. Sexual abuse of children: current concepts. $A m \mathcal{F}$ Dis Child 1982;136:142-6.

3 DeJong AR, Emmett G, Hervada AR. Sexual abuse of children: sex-,race-, and age-dependent variations. $A m \mathcal{F}$ Dis Child 1982;136:129-34.

4 Grant JJ. Assessment of child sexual abuse: eighteen months' experience at the child protection center. $A m f$ Obstet Gynecol 1984;148:617-20.

5 Schwarcz SK, Whittington WL. Sexual assault and sexually transmitted diseases: detection and management in adults and children. Rev Infect Dis 1990;12:S682-9.

6 Centers for Disease Control. 1998 sexually transmitted diseases treatment guidelines. MMWR 1998;47:RR1-116.

7 DeJong A. Sexually transmitted diseases in sexually abused children. Sex Transm Dis 1986;13:123-6.

8 Ingram DL, Everett VD, Lyna PR, et al. Epidemiology of adult sexually transmitted disease agents in children being evaluated for sexual abuse. Pediatr Infect Dis $\mathcal{F}$ 1992;11 945-50.

9 Siegel RM, Schubert CJ, Myers PA, et al. The prevalence of sexually transmitted diseases in children and adolescents evaluated for sexual abuse in Cincinnati: rationale for limited STD testing in prepubertal girls. Pediatrics 1995;96: 1090-4.

10 Ingram DL, Everett VD, Flick LAR, et al. Vaginal gonococcal cultures in sexual abuse evaluations: evaluation of selective criteria for preteenaged girls. Pediatrics 1997;99:8-16

11 American Academy of Pediatrics Committee on Child Abuse and Neglect. Guidelines for the evaluation of sexual abuse of children. Pediatrics 1991;87:254-60.

12 Hammerschlag MR. Sexually transmitted diseases in children. In: Aronoff SC, Hughes WT, Kohl S, et al, eds. Advances in pediatric infectious diseases. Chicago: Yearbook Medical Publishers, 1988;3:1-18.

3 Centers for Disease Control. Ten leading nationally notifiable infectious diseases-US. MMWR 1996;45:883-4.

14 Hammerschlag MR. Chlamydial infections. I Pediatr 1989;114:727-34

15 Hammerschlag MR. Chlamydia trachomatis in children. Pediatr Ann 1994;23:349-53.

16 Bell TA, Stamm WE, Wang SP, et al. Chronic Chlamydia trachomatis infections in infants. $7 A M A 1992 ; 267: 400-2$.

17 Hammerschlag MR, Rettig PJ, Shields ME. False positive results with the use of chlamydial antigen detection tests in the evaluation of suspected sexual abuse in children. Pediat Infect Dis F 1988;7:11-14.

18 Hauger SB, Brown J, Agre F, et al. Failure of MicroTrak to detect Chlamydia trachomatis from the genital tract of prepubertal children at risk for sexual abuse. Pediatr Infect Di f 1988; 7:660-1.

19 Porder K, Sanchez N, Roblin PM, et al. Lack of specificity of Chlamydiazyme for detection of vaginal chlamydial infection in prepubertal girls. Pediatr Infect Dis f 1989;8:358-60.

20 Centers for Disease Control. False-positive results with the use of chlamydia tests in the evaulation of suspected sexual use of chlamydia tests in the evaulation of suspec

21 Black CM. Current methods of laboratory diagnosis of Chlamydia trachomatis infections. Clin Microbiol Rev 1997, 1:160-84.
22 Schachter J. DFA, EIA, PCR, LCR and other technologies: what tests should be used for diagnosis of chlamydia infections? Immun Invest 1997;1-2:157-61.

23 Pasternack R, Vuorinen P, Pitkajarvi T, et al. Comparison of manual Amplicor PCR, Cobas Amplicor PCR and LCx assays for detection of Chlamydia trachomatis infection in women by using urine specimens. f Clin Microbiol 1997;2: $402-5$.

24 Chernesky MA, Chong S, Jang D, et al. Ability of commercial ligase chain reaction and PCR assays to diagnose Chlamydia trachomatis infections in men by testing first-void urine. F Clin Microbiol 1997;4:982-4.

25 Embree JE, Lindsay D, Williams T, et al. Acceptability and usefulness of vaginal washes in premenarcheal girls as a diagnostic procedure for sexually transmitted diseases. Pediatr Infect Dis 7 1996;8:662-7.

26 Peterson EM, Darrow, V, Blanding J, et al. Reproducibility problems with the Amplicor PCR Chlamydia trachomatis test. F Clin Microbiol 1997;4:957-9.

27 Bauwens JE, Gibbons MS, Hubbard MM, et al. Chlamydia pneumoniae (strain TWAR) isolated from two symptomfree children during evaluation for possible sexual assault. $\mathcal{F}$ Pediatr 1991;119:591-3.

28 Pate MS, Hook WE. Laboratory to laboratory variation in Chlamydia trachomatis culture practices. Sex Transm Dis 1995;5:322-6.

29 Waugh JR. Acquired syphilis of infancy and childhood. Am F Syph Gon Vener Dis 1938;22:607-22.

30 Ginsberg CM. Acquired syphilis in prepubertal children. Pediatr Infect Dis 1983;2:232-4.

31 Horowitz S, Chadwick DL. Syphilis as a sole indicator of sexual abuse: two cases with no intervention. Child Abuse Neglect 1990;14:129-32.

32 Aloi F. Lip syphilitic chancre in a child. Pediatr Dermato 1987;4:63.

33 Lande MB, Richardson AC, White KC. The role of syphilis serology in the evaluation of suspected sexual abuse. Pediat Infect Dis F 1992;11:125-7.

34 Pandhi RK, Khanna N, Sekhri R. Sexually transmitted diseases in children. Indian Pediatrics 1995;32:27-30.

35 Bays J, Chadwick D. The serologic test for syphilis in sexually abused children. Adolesc Pediatr Gynecol 1991;4:14851.

36 Moscicki AB, Palefsky J, Gonzales J, et al. Human papillomavirus infection in sexually active adolescen females: prevalence and risk factors. Pediatr Res 1990;28 507-13.

37 Fisher M, Rosenfeld WD, Burk RD. Cervicovaginal human papillomavirus infection in suburban adolescents and young adults. $\mathcal{F}$ Pediatr 1991;119:821-5.

38 Fife KH, Rogers RE, Zwickl BW. Symptomatic and asymptomatic cervical infections with human papilloma-virus tomatic cervical infections with human papil
during pregnancy. F Infect Dis 1987;156:904-11.

39 Rando RF, Lindheim S, Hasty L, et al. Increased frequency of detection of human papillomavirus deoxyribonucleic acid in exfoliated cervical cells during pregnancy. $A m \mathscr{J}$ Obstet Gynecol 1989;161:50-5.

40 Watts DH, Koutsky LA, Holmes KK, et al. Low risk of perinatal transmission of human papillomavirus: results from a prospective cohort study. Am f Obstet Gynecol (in press).

41 Sedlacek TV, Lindheim S, Eder C, et al. Mechanism for human papillomavirus transmission at birth. Am $\mathcal{F}$ Obstet Gynecol 1989;161:55-9.

42 Fife KH, Bubalo F, Boggs DL, et al. Perinatal exposure of newborns to HPV: detection by DN amplification UCLA Symposia on Molecular and Cellular Biology 1990;124:73-6.

43 Pakarian F, Kaye J, Cason J, et al. Cancer associated human papillomaviruses: perinatal transmission and persistence. Br f Obstet Gynaecol 1994;101:514-17.

44 Puranen M, Yliskoski M, Saarikoski S, et al. Vertical transmission of human papillomavirus from infected mothers to their newborn babies and persistence of the virus in childhood. Am 7 Obstet Gynecol 1996;174:694-9.

45 Cason J, Kaye JN Jewers RJ, et al. Perinatal infection and persistence of human papillomavirus types 16 and 18 in persistence of human papillomavirus

46 Shah K, Kashima H, Polk BF, et al. Rarity of cesarean delivery in cases of juvenile-onset respiratory papillomatosis. Obstet Gynecol 1986;68:795-9.

47 Gibson PE, Gardner SD, Best SJ. Human papillomavirus types in anogenital warts of children. Med Virol 1990;30 $142-5$

48 Obalek S, Jablonska S, Favre M, et al. Condylomata acuminata in children: frequent aassociation with human papillomaviruses responsible for cutaneous warts. $\mathcal{F}$ Am Acad Dermatol 1990;23:205-13.

49 Padel AF, Venning VA, Evans MF, et al. Human papillomaviruses in anogenital warts in children: typing by in situ hybridisation. BMf 1990;300:1491-4.

50 Gutman LT, St Claire KK, Herman-Giddens ME, et al. Evaluation of sexually abused and nonabused young girls for intravaginal human papillomavirus infection. Am $\mathcal{F} \mathrm{D} i$ Child 1992;146:694-9.

51 Gutman LT, St Claire KK, Everett VD, et al. Cervicalvaginal and intraanal human papillomavirus infection of young girls with external genital warts. I Infect Dis 1994;170:339-44

52 Jenison SA, Yu X, Valentine JM, et al. Evidence of prevalen genital-type human papillomavirus infections in adults and children. F Infect Dis 1990;162:60-9.

53 Speigel CA. Bacterial vaginosis. Clin Microbiol Rev 1991;4: 485-502. 
54 Nugent RP, Krohn MA, Hillier SL. Reliability of diagnosing bacterial vaginosis is improved by a standardized method of gram stain interpretation. F Clin Microbiol 1991;29:297-301.

55 Schwebke JR, Hillier SL, Sobel JD, et al. Validity of the vaginal gram stain for the diagnosis of bacterial vaginosis. Obstet Gynecol 1996;88:573-6.

56 Jenny C, Hooton TM, Bowers A, et al. Sexually transmitted diseases in victims of rape. N Engl 7 Med 1990;322:713-16.

57 Glaser JB, Schachter S, Benes S, et al. Sexually transmitted diseases in postpubertal female rape victims. F Infect Dis 1991;164:726-30.

58 Gardner JJ. Comparison of the vaginal flora in sexually abused and nonabused girls. $\mathcal{F}$ Pediatr 1992;120:872-7.

59 Bartley DL, Morgan L, Rimsza ME. Gardnerella vaginalis in prepubertal girls. Am $\mathcal{f}$ Dis Child 1987;141:1014-17.

60 Ingram DL, White ST, Lyna PR, et al. Gardnerella vaginalis infection and sexual contact in female children. Child Abuse Neglect

61 Shafer MA, Sweet RL, Ohm-Smith MJ, et al. Microbiology of the lower genital tract in postmenarchal adolescent girls: differences by sexual activity, contraception, and presence of nonspecific vaginitis. $\mathcal{F}$ Pediatr 1985;107:974-81.
62 Hammerschlag MR, Cummings M, Doraiswamy B, et al. Nonspecific vaginitis following sexual abuse in children Pediatrics 1985;75:1028-31.

63 Poch F, Levin D, Levin S, et al. Modified thioglycolate medium: a simple and reliable means for detection of Trichomonas vaginalis. $\mathcal{F}$ Clin Microbiol 1996;34:2630-1.

64 Rogers MF, Caldwell MB, Gwinn ML, et al. Epidemiology of pediatric human immunodeficiency virus infection in the United States. Acta Paediatr Suppl 1994;400:5-7.

65 Gutman LT, St Claire KK, Weedy C, et al. Human immunodeficiency virus transmission by child sexual abuse. Am $\mathcal{F}$ Dis Child 1991;145:137-41.

66 Gellert GA, Durfee MJ, Berkowitz CD, et al. Situational and sociodemographic characteristics of children infected with human immunodeficiency virus from pediatric sexual human immunodeficiency virus

67 Siegel R, Christie C, Myers $M$, et al. Incest and Pneumocystis carinii pneumonia in a twelve-year old girl: a case for early human immunodeficiency virus testing in sexually abused children. Pediatr Infect Dis 1992;11: $681-2$ 